\title{
SOME VARIATIONAL PRINCIPLES FOR PROBLEMS IN HYDRODYNAMIC AND HYDROMAGNETIC STABILITY ${ }^{1}$
}

\author{
BY \\ R. C. DiPrima \\ Department of Mathematics, Rensselaer Polytechnic Institute
}

1. Introduction. A number of problems in hydrodynamic and hydromagnetic stability give rise to characteristic value problems with differential equations of high order but with constant coefficients. Examples are the sixth order problem that arises in the stability of a viscous flow between concentric cylinders rotating in the same direction when the gap between the cylinders is small compared to the mean radius [1]; the eighth order problem that arises for the same geometry but with an electrically conducting fluid and an axial magnetic field [2]; and the sixth order problem that arises in the inhibition of convection by a magnetic field [3, 4]. (See [1] and the references cited there for a more complete list of such problems.)

Since the differential equations have constant coefficients the characteristic value problems can, in theory, be solved exactly by standard methods. However in practice such methods are often laborious and unwieldy. In several cases the characteristic value problem can be formulated as a rather unusual variational problem depending upon two functions which are related by a lower order differential equation. A variational principle of this type was apparently first suggested by Pellew and Southwell [5] in their treatment of the classical Bernard problem - the instability of a layer of fluid heated from below. More recently Chandrasekhar has used this method to treat several stability problems including those mentioned earlier [1, 2, 3, 4]. Although very satisfactory results have been obtained from variational principles of this type the computations can become somewhat tedious since it is necessary to integrate a differential equation as part of the solution. Further, the variational principles are used in such a manner that an exact solution of the characteristic value problem requires the evaluation of a determinate of infinite order. Thus only approximate (though certainly satisfactory) answers are available in practice.

In this paper it is shown how these characteristic value problems can be formulated as variational problems which can be solved exactly without the necessity of integrating any differential equation. The method of solution depends primarily upon the expansion of the unknown functions in the variational principles in complete sets of appropriate orthogonal functions. It is also shown how these methods can be extended to a non-self adjoint boundary value problem by using a variational principle dependent upon both the original problem and the adjoint boundary value problem. And in Sec. 5 a method of solving the characteristic value problem by a direct series substitution in the differential equation is illustrated.

2. The Taylor problem. The problem of the stability of a viscous fluid between two infinitely long concentric rotating cylinders was first successfully investigated both

1 Received Feb. 25, 1960. This work was sponsored by the Office of Naval Research under Contracts Nonr-591(08) and Nonr-591(12). 
theoretically and experimentally by G. J. Taylor [6]. In the case that the gap between the cylinders is small compared to a mean radius, and the cylinders are rotating in the same direction, the stability problem reduces to the determination of the minimum real positive value of $T$ for all real positive $a$ for which the following boundary value problem has a solution:

$$
\begin{gathered}
\left(D^{2}-a^{2}\right)^{3} v=-a^{2} T v, \quad-\frac{1}{2}<x<\frac{1}{2}, \\
v=\left(D^{2}-a^{2}\right) v=D\left(D^{2}-a^{2}\right) v=0 \text { at } x= \pm \frac{1}{2} .
\end{gathered}
$$

Here $D$ denotes $d / d x, a$ is a dimensionless wave number, and $T$ is a dimensionless number (called the Taylor number) which depends upon the geometry and the angular velocities of the cylinders. (Set $\alpha=0$ in Eq. 10 of [7] to obtain Eq. (1). See also [1]). The boundary value problem defined by Eqs. (1) and (2) has been solved by Pellew and Southwell [5] who found that the critical Taylor number, $T_{c}$, was 1707.8 at $a_{c} \sim 3.13$.

This boundary value problem is not self-adjoint* and hence can not be expected to be represented by a variational principle in the usual manner. However if we let $Z=T^{1 / 2}$ and $i a Z u=\left(D^{2}-a^{2}\right) v$ then Eqs. (1) and (2) become

$$
\left(D^{2}-a^{2}\right)^{2} u=i a Z v, \quad\left(D^{2}-a^{2}\right) v=i a Z u
$$

and

$$
u=D u=v=0 \text { at } x= \pm \frac{1}{2} \text {. }
$$

Now the boundary value problem defined by Eqs. (3) and (4) is equivalent to the following variational problem: Let

$$
I(u, v)=\frac{1}{2} \int_{-1 / 2}^{1 / 2}\left\{\left[\left(D^{2}-a^{2}\right\rceil u\right]^{2}-(D v)^{2}-a^{2} v^{2}\right\} d x-i a Z \int_{-1 / 2}^{1 / 2} u v d x
$$

Then among all functions $u$ and $v$ which are continuous and have continuous derivatives of the fourth order and second order respectively, and satisfy the boundary conditions (4) that pair which makes $I$ stationary necessarily satisfies the differential equations (3). To prove this statement we note that the variation in $I$ due to a variation $\delta u$ in $u$ and $\delta v$ in $v$ is

$$
\begin{aligned}
\delta I=\int_{-1 / 2}^{1 / 2}\left\{\left(D^{2}-a^{2}\right) u\left(D^{2}-a^{2}\right) \delta u-i a Z v \delta u\right\} d x & \\
& -\int_{-1 / 2}^{1 / 2}\left\{D v D \delta v+a^{2} v \delta v+i a Z u \delta v\right\} d x .
\end{aligned}
$$

If $\delta I$ is to vanish for arbitrary variations $\delta u$ and $\delta v$ that satisfy the boundary conditions (4) it follows immediately after integration by parts that $u$ and $v$ satisfy the differential equations (3).

To solve this variational problem we first note that from the form of the functional $I$ and the boundary conditions (4) that the solution can be split into even and odd func-

*Briefly, the boundary value problem $L(u)=0$ on $x_{1} \leq x \leq x_{2}$, where $L$ is a differential operator, is said to be self-adjoint if for any two functions $u$ and $v$ satisfying the specified boundary conditions at $x_{1}$ and $x_{2}$ the integral from $x_{1}$ to $x_{2}$ of $v L(u)-u L(v)$ vanishes. See $[8$, p. 59] or [9, pp. 42-44] for a more detailed discussion of self-adjoint boundary value problems. 
tions about $x=0$. For the even solution we expand $u$ and $v$ in a complete set of even orthogonal functions on $-1 / 2 \leq x \leq 1 / 2$. Appropriate series are

$$
u(x)=\sum_{n=1}^{\infty} a_{n} E_{n}(x), \quad v(x)=\sum_{n=1}^{\infty} b_{n} E_{n}(x),
$$

where $E_{n}(x)=2^{1 / 2} \cos (2 n-1) \pi x$. Notice that the boundary conditions $u=v=0$ at $x= \pm 1 / 2$ are automatically satisfied by these series. The boundary conditions $D u=0$ at $x= \pm 1 / 2$ introduce the constraining condition

$$
\Gamma=\sum_{n=1}^{\infty}(-1)^{n+1}(2 n-1) a_{n}=0
$$

on the $a_{n}$. Substituting* the series for $u$ and $v$ in the expression for $I$ gives

$$
I=\sum_{n=1}^{\infty}\left\{\frac{1}{2}\left(A_{n}^{2} a_{n}^{2}-A_{n} b_{n}^{2}\right)-i a Z a_{n} b_{n}\right\},
$$

where $A_{n}=(2 n-1)^{2} \pi^{2}+a^{2}$. The $a_{n}$ and $b_{n}$ are determined by requiring that the variation of $I$ with respect to the $a_{n}$ and $b_{n}$ vanish subject to the constraint $\Gamma=0$. The constraining condition is introduced by the use of a Lagrange multiplier. Thus we set the partial derivatives of $I-\mu \Gamma$ with respect to $a_{n}$ and $b_{n}=0$. Here $\mu$ is a Lagrange multiplier. This gives two simultaneous linear non-homogeneous equations for $a_{n}$ and $b_{n}$. Solving for $a_{n}$ and substituting in the condition $\Gamma=0$ gives the following transcendental equation for $T$ as a function of $a$

$$
\sum_{n=1}^{\infty} \frac{(2 n-1)^{2} A_{n}}{A_{n}^{3}-a^{2} T}=0 .
$$

Although the series in Eq. (9) only converges like $(2 n-1)^{-2}$, the convergence can be improved quite easily by making use of the fact that the sum of the squares of the reciprocals of the odd integers is $\pi^{2} / 8$. This gives

$$
\frac{\pi^{2}}{8}+\sum_{n=1}^{\infty}\left\{\frac{(2 n-1)^{2} A_{n}}{A_{n}^{3}-a^{2} T}-\frac{1}{(2 n-1)^{2}}\right\}=0
$$

and the convergence of this series is like $(2 n-1)^{-4}$. For a given value of $a$ the roots of Eq. (10) can be conveniently found by trial and error. The smallest positive value of $T$ is 1708.1 and occurs for $a \sim 3.12$. The solution for $u$ and $v$ odd can be carried through in exactly the same manner. For this case an appropriate set of expansion functions would be $F_{n}(x)=2^{1 / 2} \sin 2 n \pi x$.

It is perhaps worth mentioning that if the Taylor problem is written in the form of Eqs. (3) and (4) rather than the original form of Eqs. (1) and (2), approximate solutions can be obtained conveniently by the Galerkin method. This method consists of expanding $u$ and $v$ in sets of complete functions (preferably orthogonal) that satisfy the boundary conditions and then requiring the error in the equations for $u$ and $v$ to be orthogonal to the expansion functions for $u$ and $v$. (For a more detailed discussion of the Galerkin method, see Chap. 4 of [10]). For the Taylor problem appropriate functions are the $E_{n}(x)$, mentioned earlier, for $v(x)$ and the set of orthonormal even functions,

*It is permissible to differentiate these series termwise. This will be discussed in Sec. 4. 
$C_{n}(x)^{*}$, satisfying $C_{n}$ and $D C_{n}=0$ at $x= \pm \frac{1}{2}$ recently tabulated by Harris and Reid [11] for $u(x)$. In particular if one term in each series is used we obtain the approximate result $T_{c}=1728$ at $a=3.12$. This result can be improved by taking more terms in the series; however $M I$ terms in each of the series for $u$ and $v$ will lead to a determinantal equation of order $2 M$ for $T$ as a function of $a$, and the exact solution will require the evaluation of determinant of infinite order. It might also be mentioned that the higher characteristic values can be computed easily from Eq. (10). In contrast in the Galerkin method, the number of terms required in the approximating series increases as the successive characteristic values are computed.

Thus for the Taylor problem it would appear to be preferable to use the methods suggested earlier rather than to use series that satisfy the boundary conditions termwise. In Sec. 5 the use of essentially the Galerkin method to solve exactly the Taylor problem as given by Eqs. (1) and (2) by using series which do not satisfy the boundary conditions termwise will be discussed. In many problems, however, the methods of this paper are not applicable. In particular, this is true for a differential equation with variable coefficients and in these cases the Galerkin method may be very appropriate. This depends primarily upon the availability of functions satisfying the necessary boundary conditions and how closely such functions can be expected to approximate the characteristic functions of the problem. (See [12] for a discussion of a particular problem).

3. The inhibition of convection by a magnetic field. The differential equation governing the instability of a layer of an electrically conducting fluid heated from below in the presence of an external magnetic field parallel to the gravitational field is

$$
\left(D^{2}-a^{2}\right)\left\{\left(D^{2}-a^{2}\right)^{2}-Q D^{2}\right\} W=-a^{2} R W, \quad-\frac{1}{2}<x<\frac{1}{2},
$$

where $D=d / d x, a$ is a dimensionless wave number, $Q$ is a measure of the magnetic field strength, and $R$, called the Rayleigh number, depends upon the temperature gradient. The boundary conditions are

$$
W=0, \quad\left\{\left(D^{2}-a^{2}\right)^{2}-Q D^{2}\right\} W=0
$$

at $x= \pm \frac{1}{2}$ and

$$
D W=0 \text { or } D^{2} W=0
$$

at a rigid or a free surface respectively. (See [3] for a derivation of this boundary value problem). The physical problem requires for a given value of $Q$ (real and positive) the determination of the minimum real positive value of $R$ for all real positive $a$ for which this boundary value problem has a solution.

The methods of Sec. 2 are applicable to this problem. First let $Z^{2}=R$, and define $U(x)$ by $i a Z U(x)=\left\{\left(D^{2}-a^{2}\right)^{2}-Q D^{2}\right\} W$, then Eq. (11) becomes

$$
\begin{gathered}
\left\{\left(D^{2}-a^{2}\right)^{2}-Q D^{2}\right\} W=i a Z U, \\
\left(D^{2}-a^{2}\right) U=i a Z W .
\end{gathered}
$$

*The functions $C_{n}(x)$ are of the form $\left(\cosh \lambda_{m} x\right) /\left(\cosh \frac{1}{2} \lambda_{m}\right)-\left(\cos \lambda_{m} x\right) /\left(\cos \frac{1}{2} \lambda_{m}\right)$, where the $\lambda_{m}$ are the positive roots of $\tanh \frac{1}{2} \lambda+\tan \frac{1}{2} \lambda=0$. 
The boundary conditions are

$$
W=U=0
$$

at $x= \pm \frac{1}{2}$ and

1. Two rigid surfaces:

$$
D W=0 \text { at } x= \pm \frac{1}{2}
$$

2. Two free surfaces:

$$
D^{2} W=0 \quad \text { at } \quad x= \pm \frac{1}{2}
$$

3. One rigid surface and one free surface:

$$
D W=0 \quad \text { at } \quad x=\frac{1}{2}, \quad D^{2} W=0 \quad \text { at } \quad x=-\frac{1}{2} .
$$

For the variational problem the appropriate functional is

$$
\begin{aligned}
I\left(C, W^{\top}\right)=\frac{1}{2} \int_{-1 / 2}^{1 / 2}\left\{\left[\left(D^{2}-a^{2}\right) W^{\top}\right]^{2}+Q(D W)^{2}-\left(D C^{\top}\right)^{2}\right. & \left.-a^{2} U^{2}\right\} d x \\
& -i a Z \int_{-1 / 2}^{1 / 2} W U d x .
\end{aligned}
$$

For Case 1 it can be shown, as was done in Sec. 2, that a necessary condition for the vanishing of the first variation in $I(U, W)$ subject to arbitrary variations in $U$ and $W$ that satisfy the boundary conditions (15) and (16a) is that $U$ and $W^{\prime}$ satisfy the differential equations (14). This problem is precisely the same as the Taylor problem except for the term $Q(D W)^{2}$ in the definition of $I$. The appearance of this term does not cause any complications and hence this case will not be discussed further.

For Case 2 the statement of the variational problem is slightly different. The boundary conditions $D^{2} W=0$ at $x= \pm \frac{1}{2}$ are natural boundary conditions. That is, among all functions $U$ and $W$ satisfying the boundary conditions (15) that set which makes $I$ stationary will necessarily satisfy the differential equations (14) and the boundary conditions $D^{2} W=0$ at $x= \pm \frac{1}{2}$. This follows immediately upon noting that

$$
\begin{aligned}
\delta I=\int_{-1 / 2}^{1 / 2}\left\{\left(D^{2}-\right.\right. & \left.a^{2}\right) W\left(D^{2}-a^{2}\right) \delta W+Q D W D \delta W-D U D \delta U-a^{2} U \delta U \\
& -i a Z(W \delta U+U \delta W)\} d x \\
= & {\left[D^{2} W D \delta W\right]_{-1 / 2}^{1 / 2}+\int_{-1 / 2}^{1 / 2}\left\{\left(D^{2}-a^{2}\right)^{2} W-Q D^{2} W-i a Z U\right\} \delta W d x } \\
& +\int_{-1 / 2}^{1 / 2}\left\{\left(D^{2}-a^{2}\right) U-i a Z W\right\} \delta U d x
\end{aligned}
$$

after integration by parts and the use of the boundary conditions. The vanishing of $\delta I$ for $\delta U$ and $\delta W$ arbitrary in $-\frac{1}{2}<x<\frac{1}{2}$ and $D \delta W$ arbitrary at $x= \pm \frac{1}{2}$ gives the desired result. The computations are particularly simple for this case and will not be discussed. Indeed, for a solution that is even about $x=0$, one can choose $W=a_{n} \cos (2 n-1) \pi x$, $U=b_{n} \cos (2 n-1) \pi x$. The boundary conditions are then satisfied and the relationship between $R, a$, and $Q$ is found by substituting in the differential equations and setting the determinant of the coefficients of $a_{n}$ and $b_{n}$ equal to zero. 
For Case 3 the variational principle is the same as in Case 2 except that the condition $D W=0$ at $x=\frac{1}{2}$ must be satisfied by the variation in $W$; the boundary condition $D^{2} W=0$ at $x=-\frac{1}{2}$ is still a natural boundary condition. Because of the boundary conditions the solution for this case can not be split into even and odd solutions. Consequently we write

$$
W(x)=\sum_{n=1}^{\infty}\left\{a_{n} E_{n}(x)+c_{n} F_{n}(x)\right\}, \quad U(x)=\sum_{n=1}^{\infty}\left\{b_{n} E_{n}(x)+d_{n} F_{n}(x)\right\},
$$

where $E_{n}(x)=2^{1 / 2} \cos (2 n-1) \pi x$ and $F_{n}^{\prime}(x)=2^{1 / 2} \sin 2 n \pi x$. The boundary conditions $W=U=0$ at $x= \pm \frac{1}{2}$ are automatically satisfied by this choice of expansion functions. The boundary condition $D W=0$ at $x=\frac{1}{2}$ introduces the constraint

$$
\Gamma_{2}=\sum_{n=1}^{\infty}(-1)^{n+1}\left\{(2 n-1) a_{n}-2 n c_{n}\right\}=0 .
$$

Substituting the series for $W$ and $U$ in the expression for $I$ and carrying out the necessary integrations gives $I$ as a function of the $a_{n}, b_{n}, c_{n}$ and $d_{n}$. The vanishing of the first variation in $I$ subject to the constraint (19) requires the vanishing of the partial derivatives"of $I-\mu_{2} \Gamma_{2}$ with respect to the $a_{n}, b_{n}, c_{n}$ and $d_{n}$. Here $\mu_{2}$ is a Lagrange multiplier. This leads to four simultaneous linear non-homogeneous equations for $a_{n}, b_{n}, c_{n}, d_{n}$ which can be solved quite easily. Substituting for the $a_{n}$ and $c_{n}$ in the condition $\Gamma_{2}=0$. gives the following transcendental equation for $R$ as a function of $a$ and $Q$

$$
\sum_{n=1}^{\infty} \frac{n^{2}\left(n^{2} \pi^{2}+a^{2}\right)}{\left\{\left(n^{2} \pi^{2}+a^{2}\right)^{2}+n^{2} \pi^{2} Q\right\}\left(n^{2} \pi^{2}+a^{2}\right)-a^{2} R}=0 .
$$

Although this series only converges like $n^{-2}$ its convergence can be improved by adding and subtracting $\pi^{2} / 6=\sum_{1}^{\infty} n^{-2}$.

Numerical computations were carried out for two cases. In the case $Q=0$ ten terms of the series were used to compute $R_{c}=1100.6$ at $a \sim 2.86$. This case was also solved by standard techniques by Pellew and Southwell [5] who found $R_{c}=1100.65$. For $Q=50, a=3.45$ fourteen terms in the series were used to determine $R=2217.4$. It is estimated the error is less than .5\%. For this case Chandrasekhar [3] gives an upperbound of 2217.6 for $R^{*}$.

4. The Taylor problem with an axial magnetic field. Consider the Taylor problem discussed in Sec. 2 with the additional complicating feature that the fluid is an electrical conductor and there is an axial magnetic field. In this case, the stability problem requires the solution of

$$
\left\{\left(D^{2}-a^{2}\right)^{2}+Q a^{2}\right\}^{2} v=-T a^{2}\left(D^{2}-a^{2}\right) v, \quad-\frac{1}{2}<z<\frac{1}{2}
$$

with the boundary conditions

$$
\left.\begin{array}{rl}
D v & =\left(D^{2}-a^{2}\right) v=0, \\
\left\{\left(D^{2}-a^{2}\right)^{2}+Q a^{2}\right\} v & =0, \quad D\left\{\left(D^{2}-a^{2}\right)^{2}+Q a^{2}\right\} v=0
\end{array}\right\}
$$

at $z= \pm \frac{1}{2}$. Here $Q$ is a measure of the magnetic field strength, $D=d / d z$ and $a$ and $T$

*In Table 3 of [3] the values of $Q$ should be divided by four and the values of $a$ should be divided by two as pointed out by Chandrasekhar on p. 1187 of [4]. 
have the same meaning as in Sec. 2. (See [2] for a derivation of Eqs. (21) and (22).) Again, for an assigned value of $Q$, we are interested in determining the minimum positive real value of $T$ as a function of $a$ (positive) for which this boundary value problem has a solution.

To formulate this problem as a variational problem, we again split the differential equation into two lower order equations. Also in this case it is convenient, though not necessary, to change the interval of integration. If we let $x=\pi\left(z+\frac{1}{2}\right), b=a / \pi$, $P=Q / \pi^{2}, Z^{2}=T / \pi^{4}$ and $i b Z u=\left\{\left(D^{2}-b^{2}\right)^{2}+P b^{2}\right\} v$, where $D$ now denotes $d / d x$, Eqs. (21) and (22) may be written as

$$
\left.\begin{array}{l}
\left\{\left(D^{2}-b^{2}\right)^{2}+P b^{2}\right\} u=i b Z\left(D^{2}-b^{2}\right) v \\
\left\{\left(D^{2}-b^{2}\right)^{2}+P b^{2}\right\} v=i b Z u, \quad 0<x<\pi
\end{array}\right\}
$$

and

$$
u=D u=D v=\left(D^{2}-b^{2}\right) v=0
$$

at $x=0$ and $\pi$. In contrast to the problems treated in Secs. 2 and 3 the above boundary value problem, even after this splitting, is not self-adjoint and hence does not have a variational principle of the type derived previously.

In order to use the methods of the calculus of variations, it is necessary to introduce the adjoint boundary value problem. To do this, we write Eqs. (23) as $L(\mathbf{u})$ where $\mathbf{u}$ is the vector $(u, v)$ and $L$ is the matrix differential operator defined by Eqs. (23). We then form the integral of $\mathbf{U} L(\mathfrak{u})$ from 0 to $\pi$ where $\boldsymbol{U}=(U, V)$. With the use of integrations by parts this integral is then written as an integral from 0 to $\pi$ of $\mathfrak{u} L_{1}(\mathbf{U})$. The adjoint differential equations are $L_{1}(\mathrm{U})=0$. The boundary conditions are determined by requiring that the terms resulting from the integrations by parts vanish. (See p. 149 of [9]). For this particular problem the adjoint problem is

$$
\left.\begin{array}{c}
\left\{\left(D^{2}-b^{2}\right)^{2}+P b^{2}\right\} U=i b Z V \\
\left\{\left(D^{2}-b^{2}\right)^{2}+P b^{2}\right\} V=i b Z\left(D^{2}-b^{2}\right) U, \quad 0<x<\pi
\end{array}\right\}
$$

and

$$
U=D U=V=D\left(D^{2}-b^{2}\right) V=0
$$

at $x=0$ and $\pi$. Notice that if $U$ and $V$ are associated with $u$ and $v$ the boundary conditions on $V$ are different and also the role of the operator $\left(D^{2}-b^{2}\right)$ is reversed.

The original boundary value problem and the adjoint boundary value problem have the following variational principle. Let

$$
\begin{aligned}
I(u, v, U, V)=\int_{0}^{\pi}\{M(u) M(U)+M(v) M(V) & +P b^{2}(u U+v V) \\
& \left.+i b Z\left(D v D U+b^{2} v U-u V\right)\right\} d x,
\end{aligned}
$$

where $M(u)$ is $\left(D^{2}-b^{2}\right) u$. Among all functions $u, v, U$, and $V$ that are continuous and have continuous derivatives through the fourth order and satisfy the boundary conditions.

$$
u=D u=D v=0, \quad U=D U=V=0
$$

at $x=0$ and $\pi$ that set which make the first variation in $I$ vanish necessarily satisfy 
the differential equations (23) and (25) and the natural boundary conditions $\left(D^{2}-b^{2}\right) v=0$ and $D\left(D^{2}-b^{2}\right) V=0$ at the end points. The proof of this variational principle is straightforward and will not be given.

The solution of this variational problem can be split into non-combining even and odd (about $\pi / 2$ ) solutions. For the even solution appropriate orthogonal series are

$$
\left.\begin{array}{ll}
u=a_{0}+\sum a_{n} \cos n x, & U=b_{0}+\sum b_{n} \cos n x, \\
v=c_{0}+\sum c_{n} \cos n x, & V=d_{0}+\sum d_{n} \cos n x
\end{array}\right\},
$$

where $\sum$ stands for summation over the even integers. Although the series for $u, v$, and $U$ can be differentiated termwise twice, this is not true for the series for $V$. To see this we note that since $V$ is even, $D V$ is odd and $D^{2} V$ is even; hence appropriate series for these functions are $D V=\sum d_{n}^{\prime} \sin n x$, and $D^{2} V=d_{0}^{\prime \prime}+\sum d_{n}^{\prime \prime} \cos n x$. To determine the relationship between the $d_{n}, d_{n}^{\prime}$ and $d_{n}^{\prime \prime}$ we integrate by parts the following expressions for $d_{n}^{\prime}$ and $d_{n}^{\prime \prime}$

$$
\begin{aligned}
d_{n}^{\prime}=\frac{2}{\pi} \int_{0}^{\pi} D V \sin n x d x=\frac{2}{\pi}\lfloor V \sin n x]_{0}^{\pi}-\frac{2}{\pi} n \int_{0}^{\pi} V \cos n x d x \\
=-n d n, \quad n=2,4,6 \ldots .
\end{aligned}
$$

and

$$
d_{0}^{\prime \prime}=\frac{1}{\pi} \int_{0}^{\pi} D^{2} V d x=\frac{1}{\pi}[D V]_{0}^{\pi}=\frac{1}{2} \beta
$$

say, and

$$
\begin{aligned}
d_{n}^{\prime \prime}=\frac{2}{\pi} \int_{0}^{\pi} D^{2} V \cos n x d x= & \frac{2}{\pi}[D V \cos n x]_{0}^{\pi}+\frac{2}{\pi} n \int_{0}^{\pi} D V \sin n x d x \\
& =\beta+n d_{n}^{\prime}=\beta-n^{2} d_{n}, \quad n=2,4,6 \cdots .
\end{aligned}
$$

Similar computations show that the series for $u, v$, and $U$ may be differentiated termwise twice. The idea of expanding a function and its derivatives in series of complete functions and then integrating backwards to obtain the relationship between coefficients is not new. For instance it was used by S. Goldstein in 1936 [13].

Once appropriate orthogonal series for $u, v, U$ and $V$ and their derivatives have been obtained the solution of the variational problem, while slightly more complicated than in the previous cases, is straight forward and hence will only be briefly described. The boundary conditions $D u=D v=D U=0$ at $x=0$ and $\pi$ are automatically satisfied by the choice of the expansion functions. The boundary conditions $u=U=V=0$ at $x=0$ and $\pi$ introduce the constraining conditions.

$$
\Gamma_{3}=a_{0}+\sum a_{n}=0, \quad \Gamma_{4}=b_{0}+\sum b_{n}=0, \quad \Gamma_{5}=d_{0}+\sum d_{n}=0 .
$$

Substituting the series for $u, v, U$ and $V$ in the expression for $I$ and making use of relationships between $d_{n}^{\prime}, d_{n}^{\prime \prime}$ and $d_{n}$ gives $I$ as a function of the $a_{n}, b_{n}, c_{n}, d_{n}$ and $\beta$. The vanishing of the first variation in $I$ subject to the constraining conditions (31) requires that the partial derivatives of $I-\mu_{3} \Gamma_{3}-\mu_{4} \Gamma_{4}-\mu_{5} \Gamma_{5}$ with respect to the $a_{n}, b_{n}, c_{n}, d_{n}$ be zero. Here $\mu_{3}, \mu_{4}$, and $\mu_{5}$ are Lagrange multipliers. This gives four simultaneous linear nonhomogeneous equations for the $a_{n}, b_{n}, c_{n}, d_{n}$ which can be solved to give these co- 
efficients as linear functions of $\mu_{3}, \mu_{4}, \mu_{5}$ and $\beta$. The fact that we do not obtain infinitely many equations is a result of using series with the appropriate orthogonality properties. Substituting for the $a_{n}, b_{n}$, and $d_{n}$ in the constraining conditions (31) and the additional necessary condition for the vanishing of $\delta I, \partial\left(I-\mu_{3} \Gamma_{3}-\mu_{4} \Gamma_{4}-\mu_{5} \Gamma_{5}\right) / \partial \beta=0$, gives four simultaneous linear homogeneous equations for $\mu_{3}, \mu_{4}, \mu_{5}$ and $\beta$. The vanishing of the determinant of the coefficients gives a four by four determinantal equation for $Z$ as a function of $b$ and $P$. This determinant reduces immediately to a two by two determinant which can be written as

$$
X_{1} X_{2}-b^{2} Z^{2} X_{3}=0,
$$

where

$$
\begin{gathered}
X_{1}=\frac{b^{2}\left(b^{2}+P\right)}{\Delta_{0}}+2 \sum \frac{B_{n}^{2}+P b^{2}}{\Delta_{n}}, \quad X_{3}=\frac{b^{2}}{\Delta_{0}}+2 \sum \frac{B_{n}}{\Delta_{n}}, \\
X_{2}=\frac{b^{4}\left(b^{2}+P\right)}{\Delta_{0}}+2 \sum \frac{B_{n}\left(B_{n}^{2}+P b^{2}\right)}{\Delta_{n}}
\end{gathered}
$$

and $B_{n}=n^{2}+b^{2}, \Delta_{0}=b^{4}\left\{\left(b^{2}+P\right)^{2}-Z^{2}\right\}, \Delta_{n}=\left(B_{n}^{2}+P b^{2}\right)^{2}-b^{2} Z^{2} B_{n}$. Although the series in $X_{2}$ only converges like $n^{-2}$ its convergence can be improved by adding and subtracting $\pi^{2} / 24=\sum n^{-2}$. Now all the series converge like $n^{-4}$ where summation is over the even integers, hence only a few terms are needed to evaluate these series accurately.

In the case $P=0$ it can be shown that this result reduces to the solution of the Taylor problem discussed in Sec. 2 . Of course in this case these series are not as convenient as those used in Sec. 2 . For the case $Q=100, a=3.35$ a sample computation was carried out using six terms in the series. The smallest positive root was found to be $T=1.759 \times 10^{4}$. It is estimated this result is correct to less than $1 \%$. Chandrasekhar [3] gives $T=1.757 \times 10^{4}$ for this case.

5. Direct series solution. Although we have used variational techniques to solve the problems discussed in the previous sections, this is not necessary. Indeed in some cases it may be more convenient to use the direct method illustrated below for the Taylor problem that was discussed in Sec. 2.

First introduce the new variables $z=\pi\left(x+\frac{1}{2}\right), b=a / \pi$, and $Z^{2}=T / \pi^{4}$ so that the boundary value problem defined by Eqs. (1) and (2) becomes

$$
\begin{gathered}
\left(D^{2}-b^{2}\right)^{2} v=-b^{2} Z^{2} v, \quad 0<z<\pi \\
v=\left(D^{2}-b^{2}\right) v=D\left(D^{2}-b^{2}\right) v=0 \quad \text { at } \quad z=0 \text { and } \pi,
\end{gathered}
$$

where $D$ now stands for $d / d z$. For the even solution (about $z=\pi / 2$ ) of Eqs. (33) and (34), we expand $v$ and its derivatives in the following series.

$$
\begin{aligned}
v & =\sum a_{n} \sin n z & & D^{4} v=\sum e_{n} \sin n z \\
D v & =\sum b_{n} \cos n z & & D^{5} v=\sum f_{n} \cos n z \\
D^{2} v & =\sum c_{n} \sin n z & & D^{6} v=\sum g_{n} \sin n z \\
D^{3} v & =\sum d_{n} \cos n z & &
\end{aligned}
$$

where $\sum$ now stands for a summation over the odd integers. This notation will be used throughout this section. Substituting these series in the differential equation (33), 
multiplying by $\sin m z, m=1,3,5, \cdots$ and integrating from 0 to $\pi$ gives

$$
g_{n}-3 b^{2} e_{n}+3 b^{4} c_{n}-b^{6} a_{n}=-b^{2} Z^{2} a_{n}, \quad n=1,3,5 \cdots .
$$

This is similar to the Galerkin method in that the error in the differential equation is required to be orthogonal to the expansion functions; however it should be noted that the series used here do not satisfy the boundary conditions termwise. Indeed although the first two boundary conditions are automatically satisfied the boundary condition $D\left(D^{2}-b^{2}\right) v=0$ at $z=0$ and $\pi$ requires

$$
\sum\left(d_{n}-n^{2} b_{n}\right)=0 .
$$

The relationships between the coefficients in the series for $v$ and its derivatives are found by integrating backwards as was done in the previous section. Thus, $b_{n}=n a_{n}$, $c_{n}=-n^{2} a_{n}, d_{n}=-n^{3} a_{n}, e_{n}=+n^{4} a_{n}$, and

$f_{n}=\frac{2}{\pi} \int_{0}^{\pi} D^{5} v \cos n z d z=\frac{2}{\pi}\left\{\left[D^{4} v \cos n z\right]_{0}^{\pi}+n \int_{0}^{\pi} D^{4} v \sin n z d z\right\}=-\frac{4}{\pi} D^{4} v(0)+n e_{n}$ or $f_{n}=\gamma+n e_{n}=\gamma+n^{5} a_{n}$ say, and $g_{n}=-n \gamma-n^{6} a_{n}$. In deriving these relationships, use has been made of the boundary conditions that $v$ satisfies. Substituting for $g_{n}, e_{n}$, and $c_{n}$ in Eq. (35) and solving for $a_{n}$ gives $a_{n}=-n \gamma /\left\{\left(n^{2}+b^{2}\right)^{3}-b^{2} Z^{2}\right\}$. Also Eq. (36), expressed in terms of the $a_{n}$ is, $\sum n\left(n^{2}+b^{2}\right) a_{n}=0$. Substituting for the $a_{n}$ gives the following transcendental equation for $Z$ as a function of $b$,

$$
\sum_{n=1,8}^{\infty} \frac{n^{2}\left(n^{2}+b^{2}\right)}{\left(n^{2}+b^{2}\right)^{3}-b^{2} Z^{2}}=0 \text {. }
$$

This equation is identical with Eq. (9) derived in Sec. 1 using a variational procedure.

It should be noted that $a_{n} \sim n^{-5}$ and hence the solution of the characteristic value problem can be differentiated termwise four times. This result is in contrast to the remark made by Jeffreys that the trigonometric series obtained by differentiating more than twice are divergent [18]. Further a simple computation shows that $f_{n} \sim n^{-2}$ and hence the series for $D^{5} v$ and $D^{6} v$ are convergent.

6. Remarks. In the previous sections, general methods for solving exactly constant coefficient characteristic values problems have been illustrated. The methods depend primarily on the expansion of the unknown function and its derivatives in sets of appropriate orthogonal functions. It is not clear that a general conclusion can be drawn about the advantages or disadvantages of the variational method as used in Secs. 2, 3, and 4 compared to the direct method illustrated in Sec. 5. However it should be mentioned that if the direct method is used it is necessary to enforce all the boundary conditions even if some of these boundary conditions are natural boundary conditions for the variational problem.

A partial list of other hydrodynamic and hydromagnetic stability characteristic value problems that can be treated easily and exactly by the methods outlined above are the inhibition of convection by a magnetic field for a layer of fluid heated from below when the magnetic field is not parallel to the gravitational field [4], the stability of a viscous flow between two concentric cylinders rotating in the same direction in the presence of a circular magnetic field when the gap between the cylinders is small [14], and the stability of liquid helium II between concentric rotating cylinders in the case 
that the cylinders are rotating in the same direction and the gap between the cylinders is small $[15,16]$.

These methods have also been used to treat problems in the vibrations of beams and several two dimensional problems in elasticity. In particular, the reader is referred to references [17] and [19].

7. Acknowledgment. The author would like to express his appreciation to Professor Bernard Budiansky for his helpful comments during the course of this work.

\section{REFERENCES}

1. S. Chandrasekhar, On characteristic value problems in high order differential equations which arise in studies of hydrodynamic and hydromagnetic stability, Am. Math. Monthly 61, No. 7, 32-45 (1954)

2. S. Chandrasekhar, The stability of viscous flow between rotating cylinders in the presence of a magnetic field, Proc. Roy. Soc. (London) A216, 293-309 (1953)

3. S. Chandrasekhar, On the inhibition of convection by a magnetic field, Phil. Mag. Ser. 7, 43, 501-532 (1952)

4. S. Chandrasekhar, On the inhibition of convection by a magnetic field II, Phil. Mag. Ser. 7, 45, 11771091 (1954)

5. A. Pellew and R. V. Southwell, On maintained convective motion in a fluid heated from below, Proc. Roy. Soc. (London) A176, 312-343 (1940)

6. G. I. Taylor, Stability of a viscous liquid contained between two rotating cylinders, Phil. Trans. Roy. Soc. A223, 289-343 (1923)

7. S. Chandrasekhar, The stability of viscous flow between rotating cylinders, Mathematika 1, 5-13 (1954)

8. L. Collatz, Eigenwerteprobleme und ihre numerische Behandlung, Chelsea Publishing Co., New York, 1948

9. B. Friedman, Principles and techniques of applied mathematics, John Wiley and Sons, New York, 1956

10. L. V. Kantorovich and V. I. Krylov, Approximate methods of higher analysis, P. Noordhoff Ltd., Groningen, The Netherlands, 1958

11. D. L. Harris and W. H. Reid, On orthogonal functions which satisfy four boundary conditions, The Astrophysical J. 3, No. 33, 429-453 (1958).

12. R. DiPrima, Application of the Galerkin method to problems in hydrodynamic stability, Quart. Appl. Math. 13, 55-62 (1955)

13. S. Goldstein, The stability of viscous fluid flow under pressure between parallel planes, Proc. Camb. Phil. Soc. 32, 40-54 (1936)

14. F. N. Edmonds, Hydromagnetic stability of a conducting fluid in a circular magnetic field, The Phys. of Fluids 1, 30-41 (1958)

15. S. Chandrasekhar and R. Donnelly, The hydrodynamic stability of helium II between rotating cylinders I, Proc. Roy. Soc. 241, 9-28 (1957)

16. S. Chandrasekhar, The hydrodynamic stability of helium II between rotating cylinders II, Proc. Roy. Soc. 241, 29-36 (1957)

17. B. Budiansky, P. C. Hu, R. W. Connor, Notes on the Lagrangian multiplier method in elastic-stability analysis, NACA TN 1558 (1948)

18. H. Jeffreys, Some cases of instability in fluid motion, Proc. Roy. Soc. (London) A118, 195-208 (1928)

19. B. Budiansky and E. T. Kruszewski, Transverse vibrations of hollow thin-walled cylindrical beams, NACA TR 1129 (1953) 